\title{
Motivational Learning and RLC Resonance Model of Consciousness
}

\author{
Charley L. Wan \\ Holicong Middle School, 2900 Township 391 Hwy, Doylestown, PA 18902, U.S.A.; charleywan2004@gmail.com
}

\begin{abstract}
This study's purpose is to understand student performance in a school environment through student motivation dispositions and their correlation with the final grades in class. To understand what drives students' motivation dispositions, the second part of the study proposed a simple model of consciousness, an analogy to a second order RLC circuit, inspired by the "resonance theory of consciousness." Concepts of the second order RLC circuit such as resonance frequency and quality factor are used to explain how to adjust personality traits to tune your consciousness "circuit" to achieve motivational learning. The belief that intelligence is improvable underlines the mechanism of intrinsic motivation and adjustment to personality traits to synchronize both the internal and external learning environment. This study focused on students taking advanced and honor classes in high school curriculum.
\end{abstract}

KEYWORDS: Psychology; Motivation Dispositions; Achievement Goal; Resonance Theory of Consciousness; Personality Traits; Growth Mindset.

\section{- Introduction}

In a learning environment, students take various paths in school to develop a passion and pursue a goal. For learning, students often have trouble comprehending information in different subjects. This causes confusion when the student is studying and affects the results in exams. ${ }^{1}$ The underlying factor of these difficulties is motivation, the driving force for students to take interest and have a passion to learn in school. ${ }^{2}$

There are two categories of motivation in humans: performance and mastery. A performance goal focuses on the demonstration of competence relative to others while a mastery goal focuses on the development of task mastery. ${ }^{3}$ Valence, an essential aspect of motivation, represents the willingness to take risks when working towards the goal, i.e., either approaching success or avoiding failure as shown in Figure 1.

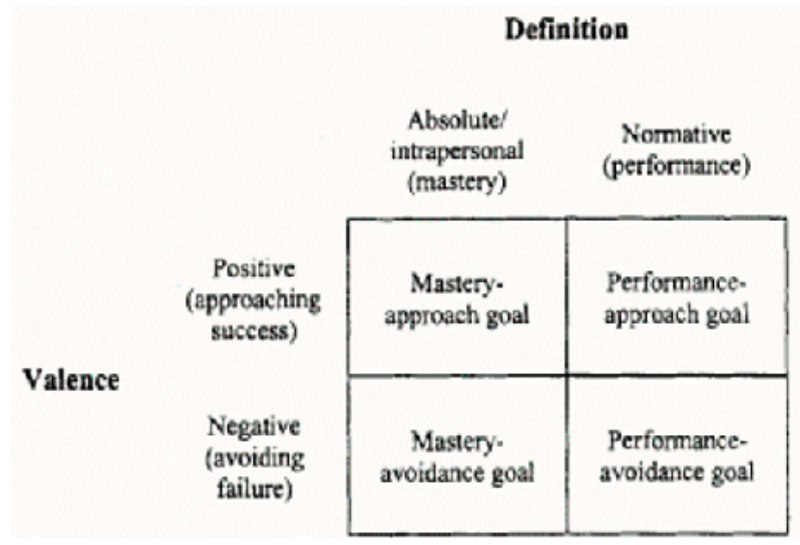

Figure 1.This matrix shows all the possible combinations of motivation a person can have based off of mastery/performance and approach/avoidance.
Generally, a student with performance motivation (including both approach and avoidance) will pick the easiest tasks, take less interest in the tasks, and give up after a failure. A student with mastery motivation will choose the most challenging tasks, enjoy the tasks, and have determination during failure. Studies on the effect of intrinsic and extrinsic motivation on children in the learning environment indicate disadvantages of performance motivation and the lack of interest or passion in the task presented. Eventually, it creates boredom and resistance to achieving the goal. ${ }^{4}$

Competence- the ability to perform successfully and efficiently - is another crucial component to the achievement of goals. Even with mastery motivation, a lack of competence decreases the likelihood of success. Therefore, both factors must be combined to create positive outcomes, represented in Figure 2. One of the most consistent patterns emerging from the achievement goal literature is the positive relationship between mastery goals and intrinsic motivation. ${ }^{2,5}$ Our brain can undergo reorganization and development. The belief that intelligence is improvable underlines the mechanism of intrinsic motivation and resilience to make up for the lack of competence. ${ }^{6,7} \mathrm{In}$ fact, growth mindset feeds into both motive dispositions and competence expectancies.

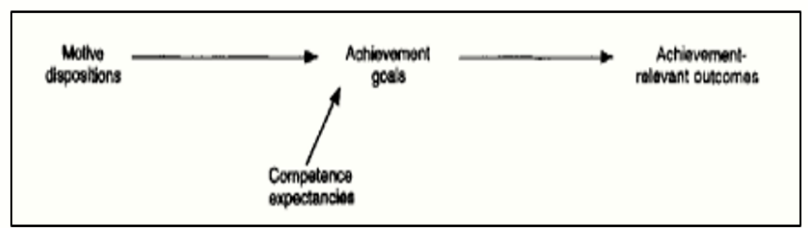

Figure 2. The achievement goal model shows the components that generate the neces sary outcomes of achievement goals . 
Intrinsic motivation naturally links the ancient "mind-body problem". The mind consists of:

- the conscious mind, a linear processor, that pays attention and decides what to do;

- the subconscious mind, a multidimensional processor in the background most of the time, doing many things simultaneously; and

- the instinctive mind, which is concerned with survival.

A metaphorical model of the mind is described as a monkey (the conscious mind), riding on an elephant (the subconscious mind) with a lizard inside (the instinctive mind), as shown in Figure $3 .^{8}$

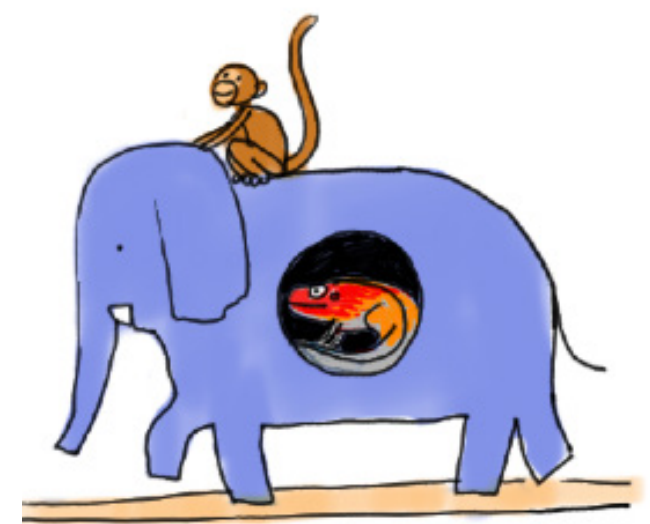

Figure 3.A visualization of the locations of each part of the mind in the metaphorical model.

The resonance theory of consciousness suggests consciousness is a form of synchronized vibrations between mind and matter. ${ }^{9}$ The theory is inspired by the phenomenon of spontaneous self-organization; when different vibrating things come together, they will eventually vibrate at the same frequency.

Consciousness is a state of mind while personality is how the mind acts. The five main personality traits are: openness to experience, conscientiousness, extraversion, agreeableness, and neuroticism. Conscientiousness is considered the strongest correlated with academic performance, but both openness and emotional stability have important associations with educational success as well. ${ }^{10}$

\section{Results and Discussion}

Empirical Correlation Between Survey: Question Scores and Grades

From the survey, students who enjoyed the class, felt fine during tests and exams, and felt satisfied about their performance obtained higher final grades than those who were not interested, tense when doing work, and unsatisfied (Figure 4). The three variables - stress, enjoyment, and perceived competence - had the strongest correlations to the final scores and were therefore used in the empirical equation. These variables determined the values of the mastery approach and perceived competence for this experiment. They also were used to predict the student's final grades. The predicted grades and actual grades are compared in Figure 5.

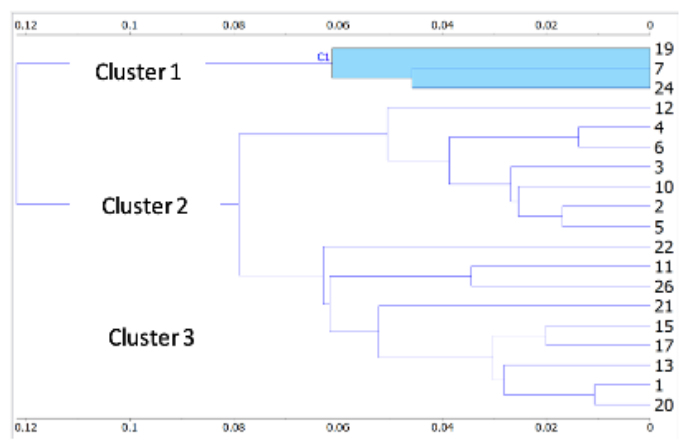

Figure 4The clustering graph shows the similarities of the survey answers. The clusters represent the groups of students that stand around the same dispositions. The numbers on the right represent student IDs.

The empirical equation is:

Final grade $=85.9+0.002 *\left(\mathrm{X}_{1}^{2} * \mathrm{X}_{2}\right)+0.67 * \mathrm{X}_{3}, \mathrm{R}^{2}=84 \%$ where

- $\mathrm{X}_{1}$, (7- magnitude of enjoyment) represents the motivation disposition of mastery or performance

- $\mathrm{X}_{2}$, (7- magnitude of stress) represents the motivation disposition of approach or avoidance

- $\mathrm{X}_{3}$, (magnitude of perceived competence) represents the competence value.

The values obtained from the equations are shown in Figure 5 .

The empirical equation coefficients were 85.9, 0.002, and 0.67, shown in Figure 6. The first is the intercept, representing the final grade a student would receive without any motivation.

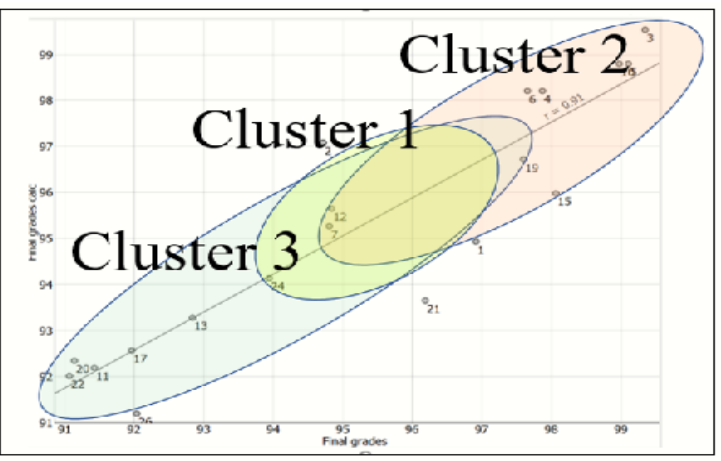

Figure 5. This graph shows a regression and clustering of the student's final grades and calculated final grades based off of the empirical equation. Cluster 2 is top right, cluster 1 is middle, and cluster 3 is bottom left.

\begin{tabular}{|c|c|c|}
\hline \multicolumn{2}{|c|}{ Regression Statistics } & \\
\hline Multiple R & 0.908609742 & \\
\hline R Square & $0.8255 / 1663$ & \\
\hline Adjusted R Square & 0.803768121 & \\
\hline Standard Error & 1.294007963 & \\
\hline \multirow[t]{2}{*}{ Observations } & 27 & \\
\hline & Coefficients & P-value \\
\hline Intercept & 85.6815644 & $4.4122 E-21$ \\
\hline Motive Dispositions & 0.002564862 & 0.00406325 \\
\hline Percieved Competence & 0.672958996 & $8.8644 E-05$ \\
\hline
\end{tabular}

Figure 6. Regression statistics consolidation of data from both the student answers from the survey and also their final grades. 
The second is the final grades' rate of change with respect to motive dispositions. The third is the final grades' rate of change with respect to perceived competency. Using these components, the accuracy of the calculated final grades to the actual final grades was $84 \%$. The regression statistics are visualized in Figure 5.

RLC Model of Consciousness and Adjusting Personality Traits for Motivational Learning

The correlation in part one highlights the importance of motivation. By giving students a survey similar to the one used in this study and implementing the practical equation teachers can understand the students' motivations and help guide them to proactively improve their motivations and ultimately their grades. However, the question remains about how to improve a student's intrinsic motivation.

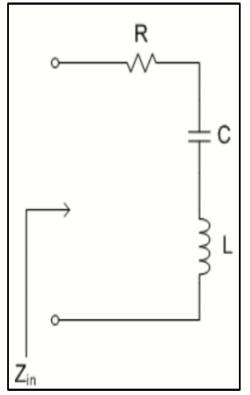

Figure 7. A diagram of the RLC circuit, the charge enters through $Z_{\text {in }}$.

Inspired by the resonance theory of consciousness, I propose a second-order serial RLC circuit model of consciousness consisting of a resistor $(\mathrm{R})$, a capacitor $(\mathrm{C})$, and an inductor (L) as shown in Figure 7. This circuit represents the brain and how the conscious mind is like a series of vibrations that can be synced up to the external world. Like the RLC circuit, the mind can filter out different vibration frequencies. This way, students can adjust themselves to a better learning style as well as coordinate between the conscious and subconscious mind. The RLC has two energy storage elements: the inductor, which stores electromagnetic energy at high frequencies, and the capacitor, which stores potential energy at low frequencies. The two energy storage elements act like a pendulum and exhibit resonance with frequency $\omega_{0}=\frac{1}{\sqrt{l C}}$ and quality factor (the sharpness of resonance) $Q_{s}=\frac{\omega_{0} L}{R} \cdot 11$

In a classroom, a student must filter out various input frequencies to sync up with the teacher. For example, if the teacher's input is fast paced then the student should apply the high-pass filter (the instinctive/lizard mind) to sync up with the teacher. If the teacher's input is medium paced the student should apply the band-pass filter (the conscious/monkey mind). If the teacher's input is slow-paced the student should apply the low-pass filter (the subconscious/elephant mind). This example is displayed in Figure 8.

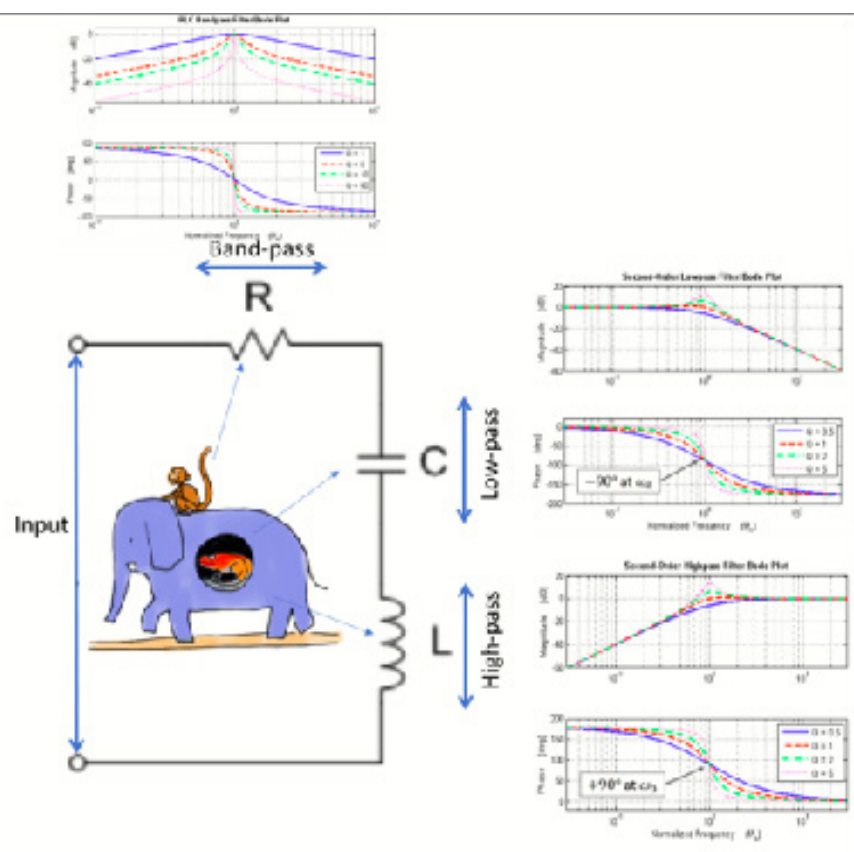

Figure 8. An analogy of the metaphorical model of the mind to the RLC circuit. The circuit frequencies are waves that have three segments: the low, high and band pass. This represents different working environments where transitions between different levels of concentration are required to sync up and extract important information. It also represents the communication between the elephant, monkey and lizard or the subconscious, conscious and instinctive mind, respectively.

The design of an RLC circuit for signal filtering depends on selection of circuit impedances for the resistor, capacitor, and inductor. Similarly, a student's ability to sync up their internal conscious and subconscious minds, and the internal mind to the external world depends on adjusting their personality traits towards motivational learning..

An RLC filter can be designed to give any resonance frequency $\omega_{0}=\frac{1}{\sqrt{x}}$ by specifying the impendence of the inductor and capacitor.

Similarly, each main personality trait is between two extremes (similar to the highest and lowest points of a pendulum), with one gaining energy at higher frequencies and the other gaining energy at lower frequencies.

- Openness to experience inventive/curious (high) vs. consistent/cautious (low)

- Conscientiousness efficient/organized (high) vs. easy-going/careless (low)

- Extraversion outgoing/energetic (high) vs. solitary/reserved (low)

- Agreeability friendly/compassionate (high) vs. challenging/detached (low)

- Neuroticism sensitive/nervous (high) vs. secure/confident (low)

To sync one's conscious mind (the monkey, which tends to operate at higher frequencies and seek performance scores) and subconscious mind (the elephant which tends to operate at lower frequencies and seek mastery experience), students must swing between the personality extremes so that both minds can be fully utilized. 
Likewise, to sync one's internal mind with the external world the student should be more curious, efficient, energetic, compassionate, and sensitive if the teacher's style is fast paced while a slow-paced teacher requires students' to be cautious, easy-going, reserved, detached and secure.

In the survey, students were asked about their extracurricular activities. Students who enrich their curriculums with extracurriculars make more connections with and receive benefits from their subconscious mind. They are more likely to achieve higher performance scores. Prior research supports that increased involvement within the school community is correlated with higher performance achievement. ${ }^{12}$ The experimental relationship between extracurricular activities and final grades is depicted in Figure 9. As Professor Little said in his 2016 TED talk, to achieve our core personal projects (such as professions), we can "act out of characters" (personality traits), and that is what makes us unique. ${ }^{13,14}$

Final grades vs No. of extracurricular activities

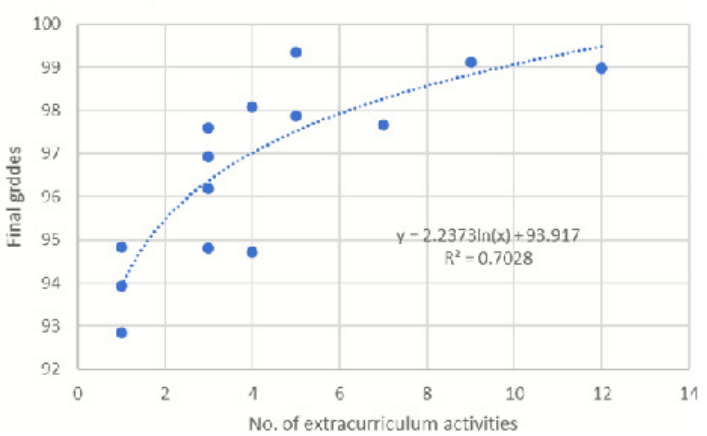

Figure 9. The relationship between the number of extracurricular activities and the final grade for each student surveyed.

\section{Discussion}

The structure of the mind oversees the motivation and achievement processes. Another way of interpreting the mind is by using the analogy of homework, lessons, and exams. The lesson is like the elephant; it contains the most imperative information and stores it in the subconscious. The daily homework is like the monkey; only the information that is quickly memorized and temporarily retained is stored in the conscious mind. Finally, when all the knowledge has built up, an exam is given. Since the mind has limits to its retention of important information, many students are nervous during final exams. This is much like how the lizard part of the mind activates fight or flight responses during stressful events. Education systems that implement creative and novel ways to teach students-such as year-long projects-allow a steady flow of knowledge and challenge. This generates a better system of communication between the three parts of the mind and cultivates intrinsic motivation.

Student intrinsic motivation comes from how the mind reacts to different experiences relating to one's personality. The reason students have trouble doing well with specific classes could be attributed to the conjecture that they are unable to sync their internal conscious and subconscious minds collectively or sync their internal minds to the external educational world. By adjusting their personalities as suggested, students and teachers together may create a better learning experience and produce better performance.

\section{Conclusion}

This experiment mainly focuses on the significance of moti vation for students in a school environment. The study shows a clear connection between survey results and student scores. If the student answers swayed towards positive qualities (i.e., high enjoyment, low stress, high competence) the final grade received in the science class was higher. The experiment yielded a practical equation to predict grades based on the survey answers.

To find practical actions for improving intrinsic motivation, a simple model of consciousness was proposed, inspired by the resonance theory of consciousness. This second order RLC circuit model has two energy storage elements, representing the conscious and subconscious as well as the two extremes of personality. Personality adjustments can filter desired information from the external world into the internal mind and improve intrinsic motivation and achievement.

For future research, surveys should be given to the teachers and students to measure the similarities and differences in their personalities. Therefore, adjustments can be made to minimize differences to sync up students with the teacher. Surveys would then be given to students and put into different environments (i.e., the classroom or at home) to measure the similarities and differences in their personality. This research structure is illustrated in Figure 10. The results could then be analyzed in the perspective of the student and show their interactions between the conscious and subconscious mind. In the above research cases, the independent variables would be the survey scores. The dependent variable would be intrinsic motivation. This is an extension of current research, where intrinsic motivation is the independent variable and achievement goals (grades) is the dependent variable.

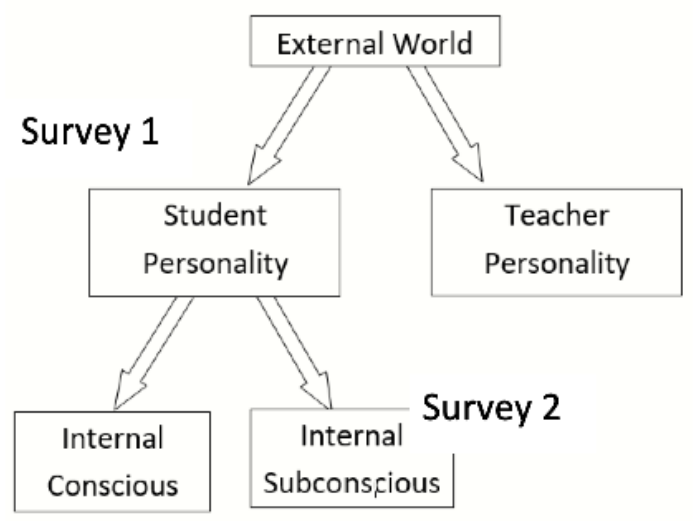

Figure 10. A representation of the future research concept. The first survey is for the teachers and students to understand their personalities in the learning environment. The second survey is for students to understand their personalities in different environments.

\section{Methods}

Survey participants were 9th graders aged $14-15$ at Holicong Middle School. They had taken either advanced or academic science in $8^{\text {th }}$ grade. Students were given consent 
forms to agree to the experiment. Students who agreed were given the survey through confirmation of the principal. The surveys were comprised of questions chosen from the Intrinsic Motivation Inventory with two questions from each category. ${ }^{13}$

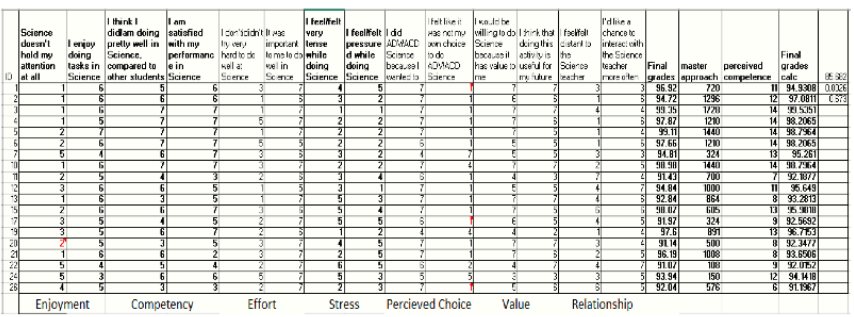

Figure 11. Survey answer spreadsheet, most important variables are enjoyment, competency and stress; the last three columns represents results from the empirical equation to calculate approximate final grades for the students using the three variables and coefficients.

The students answered on a 1-7 scale similar to the Likerttype evaluation as shown in Figure 11. The survey set contained 19 students with $60 \%$ advanced class and $40 \%$ academic class representatives. The answers to the survey are displayed in Figure 11 . The clustering method was used for the survey analysis to understand the general motive dispositions among the set of students. Final grades of students were also used to yield the results of this experiment. Given permission by the school principal, grades were analyzed based on the performance of the students the year they took the science classes, $8^{\text {th }}$ grade, using regression.

\section{Acknowledgements}

I would like to thank my parents, for supporting my experiment. I also thank my science teacher, Mr. Meletti, and principal, Dr. Shillingford, who supported the success of the data collection and analysis with key advice that wound up as a full extension to the Resonance Theory of Consciousness with RLC modelling.

\section{References}

1. Kusurkar, R. A.; Ten Cate, T. J.; Vos, C. M. P.; Wasters, P.; Croiset, G. How motivation affects academic performance: a structural equation modelling analysis. Adv. Health Sc.i Educ. Theory Pract. 2012, 18(1), 5769. https://www.ncbi.nlm.nih.gov/pmc/articles/PMC3569579/ (accessed May 8, 2020).

2. Ryan, R. M.; Deci, E. L. Self-determination theory and the facilitation of intrinsic motivation, social development, and well-being. Am. Psychol. 2000, 55(1), 1-11. https://psycnet.apa.org/doi/10.1037/0003-066X.55.1.68

3. Elliot, A. J.; Church M. A. A Hierarchical Model of Approach and Avoid ance Achievement Motivation. J. Pers. Soc. Psychol. 1997, 72(1), 1-15.

4. Luskin, C. V. Mark Lepper: Intrinsic Motivation, Extrinsic Motivation and the Process of Learning. The Bing Times, Sep 2003, pp. 5-7.

Retrieved from bingschool.stanford.edu/news/mark-lepper-intrinsic motivation-extrinsic-motivation-and-process-learning (accessed Mar 7, 2020).

5. Elliot, A. J.; McGregor, H. A. A 2 × 2 achievement goal framework. J. Pers. Soc. Psychol. 2001, 80(3), 1-19.

6. $\mathrm{Ng}, \mathrm{B}$. The Neuroscience of Growth Mindset and Intrinsic Motivation. Brain Sci. 2018, 8(2), 1-10. https://link.springer.com/article/10.1023/A:1026712428094

7. Dweck, C. S.; Yeager, D. S. Mindsets: A View from Two Eras. Perspect. Psychol. Sci. 2019, 14(3), 1-45.

8. Gray, D. A Metaphorical Model of the Mind. Medium, 25 Mar 2016. Retrieved from medium.com/the-overlap/a-metaphorical-model-of- the-mind-f3b243ce787b. (accessed 7 Mar 2020).

9. Hunt, T. Kicking the psychophysical laws into gear a new approach to the combination problem. J. Conscious, Stud. 2011, 18(11), 96-134.

10. Vedel, A.; Poropat, A. Personality and Academic Performance. Encyclopedia of Personality and Individual Differences [Online]; Springer, Cham, Posted Feb 1, 2017. https://doi.org/10.1007/978-3-319-28099-8

11.RLC Circuit. Wikipedia, Apr 26, 2020, https://en.wikipedia.org/ wiki/RLC_circuit (accessed May 10, 2020).

12. O'Brien, E.; Rollefson, M. Extracurricular Participation and Student Engagement, 1995. National Center for Education Statistics website. https://nces.ed.gov/pubs95/web/95741.asp (accessed May 9, 2020).

13. Little, Brian (2016) "Who are you, really? The puzzle of personality" https://www.youtube.com/watch?v=qYvXk_bqlBk

14. Little, Brian R. \&quot;Personal Projects, Happiness, and Meaning: On Doing Well and Being Yourself.\&quot; Personal Projects, Happiness, and Meaning: On Doing Well and Being Yourself., PDF

\section{Author}

Charley L. Wan is a $9^{\text {th }}$ grade at Holicong Middle School. This experiment was inspired by his experience as a teenager and his curiosity about his peers' experience in school. He is very interested in tinkering with new problems of math and science. 